\title{
Surface modification of anhydrous borax powders with stearic acid via mechanical dry powder coating
}

\author{
Süleyman Akpınari*, Zeyni Arsoy², R. Sena Şenol ${ }^{3}$ \\ ${ }^{1}$ Afyon Kocatepe University, Department of Material Science and Engineering, Afyonkarahisar, Turkey, \\ ORCID ID orcid.org/0000-0002-7959-3464
}

${ }^{2}$ Afyon Kocatepe University, Department of Mining Engineering, Afyonkarahisar, Turkey, ORCID ID orcid.org/0000-0001-5694-6338 ${ }^{3}$ Afyon Kocatepe University, Department of Material Science and Engineering, Afyonkarahisar, Turkey, ORCID ID orcid.org/0000-0003-2028-2335

\section{ARTICLE INFO}

Article history:

Received 11 April 2018

Revised form 04 December 2018

Accepted 10 January 2019

Online 16 March 2019

Research Article

DOI: $10.30728 /$ boron.414410

\section{Keywords:}

Anhydrous borax,

Dry coating,

Solubility,

Stearic acid,

Surface modification

\begin{abstract}
Surface modification of anhydrous borax powders with stearic acid in the laboratoryscale planetary ball mill via mechanical dry powder coating approach was investigated. The alteration of hydrophilic surface properties of anhydrous borax with stearic acid was optimized by modifier amount and activation time. The cohesion mechanisms of powders, which processed at conditions of $0.5,1$ and 2 wt.\% stearic acid amount with the function of 30,60 and 120 minute activation time, were evaluated in terms of the solubility test, contact angle measurements, particle size distributions, and scanning electron microscopy (SEM) analysis. Results indicate that anhydrous borax surface could be switched from hydrophilic to hydrophobic with changing contact angle from wetting $\left(17^{\circ}\right)$ to non-wetting $\left(9^{\circ}\right)$. Water-insoluble amount of anhydrous borax powders, as a coating efficiency indicator, was increased from $73 \%$ to $91 \%$ by coating with 1 wt.\% stearic acid for $60 \mathrm{~min}$. Furthermore, SEM analysis results demonstrate that stearic acid was discretely coated over the anhydrous borax surface. As a conclusion, an effective mechanical dry coating processing by a one-step coating approach could be applied to obtain a modified anhydrous borax surface, which offers controlled solubility behaviour in water-based suspensions.
\end{abstract}

\section{Introduction}

Boron minerals are widely utilized in numerous industrial areas such as ceramic, glass, cement, metallurgy, agriculture, medicine, cosmetics, automotive, communication, insulation, energy, etc. [1]. There is a growing interest in widening the application fields of boron and its derivates, due to their promising novel advantages. Boron minerals are differently named regarding their alkaline, earth alkaline, boron oxide $\left(\mathrm{B}_{2} \mathrm{O}_{3}\right)$ and water contents and crystal structures. However, only some of them (tincal, colemanite, ulexite, hydroboracite, sassolite, etc.) are of commercial importance [2]. Refined borates or primary boron chemicals (borax pentahydrate, borax decahydrate, anhydrous borax, boric acid, sodium perborate, etc.) are chemically refined run of mine boron minerals $[3,4]$.

Anhydrous borax $\left(\mathrm{Na}_{2} \mathrm{~B}_{4} \mathrm{O}_{7}\right)$ is directly produced by dehydrating borax, and the most common form of this a-crystalline which melts congruently at $742.5^{\circ} \mathrm{C}$ [5]. It is an excellent fluxing agent and a glass former. Its usage improves yields and provides less energy-intensive processes for glass and ceramic industries [6]. Although it dissolves more slowly than hydrated forms in water, anhydrous borax still has significant solubility values at room temperature in the liquid media, such as 3.37 wt. \% in water, 16.7 wt. \% in methanol and 30 wt. \% in ethylene glycol [5].

The borates enter the aqueous medium most readily when used directly in the form of water-soluble inorganic borates. This property provides advantages, for example; its usage in perborate-containing detergents, boronated fertilizers, additives to corrosion inhibitors in anti-freeze formulations, biocides for cutting fluids, insecticides, and as buffers/preservatives for cosmetic and pharmaceutical preparations [7].

On the other hand, borates can dissolve into the glaze suspension, causing disadvantages such as an alteration of the glaze as it dries on the ceramic bisque due to its partial solubility. Water wicks into the interior of the bisque during application. During drying, the water containing soluble material migrates to the ridges or high areas of the form causing blistering and dry glaze areas. Besides partial solubility, it can absorb water from the atmosphere during storage because of hygroscopic nature, resulting in the possibility of inaccurate amounts being placed into a glaze formula [8]. Moreover, it negatively affects the rheological behaviours of glaze suspension as of being soluble in water. 
Powder coating is a method that focuses on improving or modifying some specific properties of powders that the natural product does not offer. Particle coating or surface modification technology has been used for the synthesis of hybrid materials with the desired properties in many industries, including pharmaceuticals, food, cosmetics, ceramics, electronics, and special chemicals. At present, most commercial powder coatings are done by wet coating methods such as solgel processes, chemical deposition, spray coating, dip coating, spinning disc coaters and a variety of fluidized bed coaters. Wet particle coating is used primarily to form a barrier or film between the host particle and its surrounding. The coating material usually consists of a solute dissolved in an organic solvent or an aqueous suspension of the solute. The organic solvent is highly volatile (VOC). VOCs have been implicated as a major precursor in the production of photochemical smog, which causes atmospheric haze, eye irritation and respiratory problems and even some VOCs are carcinogenic. These environmental drawbacks of wet coating methods have forced researchers in the powder technology field to find alternative methods for coating of powders [9].

Dry particle coating is a newly emerging and alternative approach to wet coating methods, and it has drawn the attention of many researchers. It uses mechanical forces (mechanical impact, shearing, etc.) in order to attach submicron-sized fine particles (guest) on to relatively larger micron-sized (host) particles without using any solvents, binders or even water. Dry particle coating is used to make modifications of the properties or functionality of the host particle and create a new generation of composite particles. Some examples of surface properties that can be improved or modified are flowability, dispersibility, solubility, wettability, electrostatic, electric, magnetic, optical, colour, flavour, taste, particle shape/sphericity, sinterability, and solid phase reactivity. In addition to producing materials with different functionality, dry coating process has an advantage of being cost effective due to the reduced use of high-price or rare materials since the more expensive material (guest) can be coated onto the cheaper carrier material (host) and also there is no need for drying the products, which result in substantial energy savings. Another major advantage of the dry particle coating process is that it is environmentally benign, producing none of the organic (gas or liquid) or aqueous waste streams, which usually are present in wet coating processes $[9,10]$.

In dry particle coating processes, powdered materials with a relatively large particle size (host particles; $1-500 \mu \mathrm{m})$ are mechanically coated with fine particles (guest particles; 0.1-50 $\mu \mathrm{m}$ ) in order to create new functionality or to improve their initial characteristics $[11,12]$. Since the size of the guest particles is so small, van der Waals interactions are strong enough to keep them firmly attached to the host particles. Thus, either a discrete or a continuous coating of guest particles can be achieved depending on a variety of operating conditions, including activation time, mechanical action, the weight fraction of guest to host particles, and the physical properties of the particles used $[10,12,13]$. The process of dry particle coating is illustrated in Figure 1.

There are many methods and devices used for particle dry coating, e.g. Mechanofusion, Hybridizer and Cyclomix for applying a high level of mechanical forces, or Magnetically Assisted Impaction Coater (MAIC), Theta Composer, V-Blender and Rotating Fluidized Bed Coater (RFBC) for applying a small level of mechanical forces. There are many applications of the dry particle coating process in different fields. $\mathrm{TiO}_{2}$ coating of polymethyl methacrylate (PMMA) particles using mechanofusion to improve the flowability, cellulose coating of silica using MAIC and food fibre sorbitol + glucose coating of hydrophilic silica using theta composer for modification of flowability and water affinity, polyethylene coating of hydroxyapatite using hybridizer for evaluation of separation capacity of composite proteins, magnesium stearate coating of silica gel using hybridizer and cyclomix for modification of flowability and wettability [9]. The operating principles of each of the coating devices referred to above are different; hence, the type of coating produced and the applications for which they are optimum are also different. Coatings can be characterized into several categories
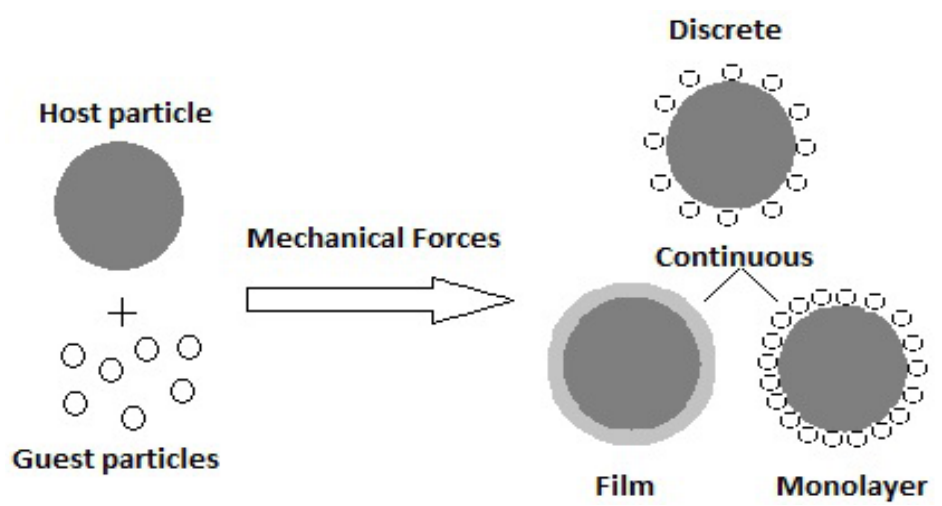

Figure 1. Dry particle coating principle [12]. 
such as deep embedding, encapsulation, filming, discrete or partial surface covering, and a loose surface coating (ordered mixture) [10]. A planetary ball mill was used as an alternative promising tool by Sonoda et al. 2008 [14], to coat starch particles with a poorly watersoluble drug-flurbiprofen. The planetary ball milling device operates in the centrifugal mode, which leads to a more gentle size reduction process with fewer abrasions. Besides, the difference in speeds between the balls and the grinding medium produces high-impact forces. These forces provide the possibility of very efficient mixing and coating in the planetary ball mill [1517]. The subject of dry particle coating is very closely related to the subject of dry mixing of powders [10].

Actually, under ordered mixing of a binary blend of fine and coarse particles, the fine particles display high levels of cohesivity, which can lead to the formation of agglomerates or segregation, producing non-homogeneous mixtures $[18,19]$. When the powders are cohesive, the mixing two constituents require breaking-up of the agglomerates. When the constituents differ in size there is an increased tendency for segregation as the size difference becomes larger. However, when the two components to be mixed are very different in size, then segregation may no longer be a problem. In such cases, the smaller particles tend to adhere on to the larger particles. The adhesion force between the smaller particle and the larger particle is greater than the weight of the smaller particle, and hence it is not easily removed from the host. Dry particle coating overcomes the high cohesive forces associated with the fine "guest" particles and thus becoming uniformly bound to surface of coarse "host" particles by strong forces of adhesion $[10,19]$. In ordered mixing, the surface of the larger particles is loosely coated/covered with smaller particles. In dry particle coating, the same thing happens; however, the surface covering is more permanent because of a stronger physical (or chemical) bonding [10]. Therefore, any mixing device, in order to achieve ordered mixing, must provide sufficient mechanical or other means of agitation to promote de-agglomeration of fines, i.e., provide a large number of particle collisions involving high normal and shear impact forces. Machines that can be used for this purpose are high-intensity mixers and grinding machines such as ball and media mills [10].

According to the relevant literature, the fundamentals of dry coating studies with stearic acid as the guest particle; a variety of inorganic powders (such as calcite [20-22] and magnesium hydroxide $[20,23]$ etc.) is selected as host particles. These coating processes were performed in various mixers. The common features of these devices are high rotational speed providing and the probability of particle collision, and mechanofusion effect. However, it has been determined that the analog behaviour of these processes is; having an effective activation time for each device. It is important to reveal an optimal time for the coating process since the host particle's coverage efficiency fades away when this period exceeds. The further resemblance in researches; analytical methods such as X-ray photoelectron spectroscopy (XPS) and environmental scanning electron microscopy (ESEM) etc., and physical measurements such as contact angle measurement, flowability, and active index were applied for the determination of coating success. The improved surface properties of the final products have not only evaluated with wettability but also improvement of other physical properties such as solubility reduction and powder flowability increment, etc.

Fatty acids contain the acidic $(-\mathrm{COOH})$ group. They have the general formula $\mathrm{R}-\mathrm{COOH}$ where the side chain, $\mathrm{R}$, is hydrogen or an alkyl group, such as $-\mathrm{CH}_{3}$ or $-\mathrm{C}_{2} \mathrm{H}_{5}$, and so on. They increase in molecular length by $-\mathrm{CH}_{2}$ for each successive member of the series. Most fatty acids have an even number of carbon atoms, usually between 14 and 22 . Fatty acid molecules are insoluble in water due to these long hydrocarbon tails, which are referred to as hydrophobic or waterhating [24].

Fatty acids frequently contain double bonds $(C=C)$ in their side chains $(R)$ resulting from the absence of some hydrogen atoms. If a fatty acid has a full complement of hydrogen atoms, so it has no double bonds, then it is said to be saturated fatty acid. The general formula of a saturated fatty acid is $\mathrm{C}_{n} \mathrm{H}_{2 n}+1 \mathrm{COOH}$. Examples are palmitic acid $\left(\mathrm{C}_{15} \mathrm{H}_{31} \mathrm{COOH}\right)$ and stearic acid $\left(\mathrm{C}_{17} \mathrm{H}_{35} \mathrm{COOH}\right)$. If a fatty acid has some hydrogen atoms missing that resulting in the presence of double bonds, then it is referred to as unsaturated fatty acid [24].

Stearic acid $\left(\mathrm{C}_{17} \mathrm{H}_{35} \mathrm{CO}_{2} \mathrm{H}\right)$ is a saturated fatty acid with a melting point of $69.6^{\circ} \mathrm{C}$ [25], and containing 18 carbons [24]. This length is categorized as long-chained (>12 carbons), this chain length makes it insoluble in water [26], but this acid is soluble in ethanol [27]. Stearic acid is commonly found in animal and vegetable fats, and that is frequently used in cosmetics, candles, soaps, plastics, oil pastels and for softening rubber [28].

Stearic acid is a low-cost organic acid, and its molecule has hydrophilic and hydrophobic groups; therefore, it has been used as the mineral modifier [29]. It is generally considered that the interaction between mineral and stearic acid is physical adsorption [30]. However, some studies reported that there is chemisorption between calcium carbonate and stearic acid [31].

The present study aims to apply simple one-step powder technique in order to demonstrate the effect of mechanical dry coating on the solubility of anhydrous borax particles coated with three different weight ratio of stearic acid by using a planetary ball milling. 


\section{Materials and methods}

\subsection{Materials}

Anhydrous borax (ANB) powders of purity 99\% (ETIBOR-68) was supplied by Eti Mine Enterprises (Turkey). The particle size of the as-received ANB powders is under $500 \mu \mathrm{m}$ and the d50 value of powders is about $250 \mu \mathrm{m}$. ANB was utilized as a host particle in the dry coating, further ground to fine powder by planetary ball milling, as described below. Stearic acid (SA), as guest particles, is a fine, white, greasy and cohesive powder widely used in the pharmaceutical formulation as a lubricant. The starting material was treated with stearic acid, 0.5, 1 and $2 \mathrm{wt}$. \%, to yield different surface coverage of the anhydrous borax. The main properties (as provided by the manufacturer) of stearic acid, encoded as Wilfarin SA-1865, are presented in Table 1.

Table 1. Technical data for stearic acid.

\begin{tabular}{ll}
\hline Property & Value \\
\hline Formula & $\mathrm{CH}_{3}\left(\mathrm{CH}_{2}\right)_{16} \mathrm{COOH}$ \\
Molecular weight $(\mathrm{g} / \mathrm{mol})$ & 284.47 \\
Boiling point $\left({ }^{\circ} \mathrm{C}\right)$ & 383 \\
Melting point $\left({ }^{\circ} \mathrm{C}\right)$ & 69.08 \\
Density $\left(\mathrm{g} / \mathrm{cm}^{3}\right)$ & 0.941 \\
\hline
\end{tabular}

\subsection{Dry powder coating processes}

In the present study, a dry process as a one-step easy route was preferred for grinding, mixing and coating powders simultaneously by using a planetary ball mill. This laboratory-scale milling device has the working chamber with a jar $(500 \mathrm{~mL})$ filled with alumina balls (10-20 mm diameter) and placed on the rotating basis plate. The working chamber is attached to the basis plate which rotates around the central axis, while the chamber rotates around its own axis in an opposite direction. The ratio of the velocity of the basis plate and the jar is constant, and the ratio of the grindings balls to powder weight was like $2: 1$ and $60 \%$ void volume.

The dry coating process in the planetary ball milling system can be summarized as follows: Prior to the coating treatment, ANB (100 g) was manually premixed with SA at mass fractions of $0.5,1$, and $2 \%$. For each coating experiment, the mixtures of the powders were fed into the milling jars and processed under the following operation modes; at constant rotational speed, three different activation times and amount of guest particles. The planetary ball milling processing was performed at a rotation speed of $350 \mathrm{rpm}$ in order to coat the SA onto ANB particles by dry-grinding to a certain size for a given period. The activation time was selected to 30, 60 and 120 minute. During the process of milling the powder mixture is subjected to high impaction and dispersion due to friction with balls and the walls of the device and continuously re-circulates in the machine through the grinding jars. The material is trapped between colliding balls and between the balls and the walls of the jars which cause breaking agglomerates apart and deposition of guest particles on the surface of host particles. After being processed for the prescribed application mode, the processed powders were thoroughly removed from the milling jar and then stored in a desiccator for the subsequent characterization measurements.

\subsection{Characterization}

\subsubsection{Scanning electron microscopy and EDX analysis}

The as-received and coated ANB powders were examined by scanning electron microscopy (SEM, Leo 1430 VP) using an accelerating voltage of $20 \mathrm{kV}$ to study the surface morphology and particle shape before and after coating, and the covering by the SA particles. Double-sided carbon tape was attached to the sample holder, and the powder sample in small quantities was poured onto the adhesive surface. Excess powder was tapped and in some cases blown away gently with a manual air pump. Then, the samples were sputtercoated with a thin gold layer with BAL-TEC SCD 005 sputter coater that operated for $40 \mathrm{~s}$ at $100 \mathrm{~mA}$. Secondary electron images (SEI) were used for surface morphology examination. In addition, the existence of stearic acid coating present on the surface of the powders was detected by EDX-SEM studies.

\subsubsection{Measurement of particle size distribution}

The particle size distributions of as-received and coated ANB powders were measured using a Laser Particle Size Analyzer (BT-9300Z, Bettersize Instruments Ltd., China). $D_{10}, D_{50}$, and $D_{90}$ values were obtained from the particle size analysis and compared to the result that was manually measured using the scale bar on the SEM images. In a typical trail of manual measurement, 20 particles were taken as samples and the average value was used for further calculation. On the other hand, for comparison with as-received powders, the particle size distribution of powders coated with $1 \mathrm{wt} . \%$ SA for 60 min was measured. Three measurements were performed for each experiment, and the mean and standard deviation were calculated.

\subsubsection{Wettability measurements}

The contact angles of the powders were measured by the sessile drop method (KSV Attension ThetaLite TL 101 Optical Tensiometer) at room temperature (25 \pm 2 ${ }^{\circ} \mathrm{C}$ ) by deposing a small drop (five drops per sample) of pure water on tablets formed from powder. The tablet discs with $1.5 \mathrm{~cm}$ diameter were made by a compression under a load of $10 \mathrm{kN}$ using a uniaxial manual hydraulic press and labeled discs were kept in a desiccator until contact angle measurements. The shape of the drops was observed and used to determine the contact angles. 
The operation principle of the instrument based on the addition of a liquid with known surface tension onto the disc with a Hamilton syringe $(1 \mu \mathrm{L})$ and taking pictures of the water drop formed on the treated solid surface by a video camera. After the tip of the needle was separated from the drop, the CCD camera captured the side view of the water drop, and the contact angle was measured by a digital goniometer. 10 different pictures of the droplet are recorded in a very short time interval (millisecond) after addition of liquid on the solid surface. Finally, the contact angle of each droplet on the tablet discs is determined, and an average value is obtained by taking arithmetic mean of contact angle values. This process is repeated on five different surface locations on the disc and contact angle values are obtained for these locations. Average of these values gives the final contact angle value for that liquid.

\subsubsection{Determination of the coating efficiency and durability}

ANB as a water-soluble material has a great tendency towards hydration; therefore, insoluble SA was commissioned to prevent its water interaction. After a thorough review of the literature, it was clearly understood that determination of the coating efficiency for soluble and non-soluble materials must differ. Previously in the relevant literature, the coating efficiency was evaluated by activation index [32], which is the weight ratio of the floated part on the water to that of the overall sample for non-soluble materials. Since the mass of soluble materials differed during the activation index test, there has been a need for a new coating efficiency assessment. In conjunction, unlike the mentioned from the literature, coating efficiency was evaluated according to the change in the amount of water-insoluble ANB powders depend on process parameters.

Coating efficiency assessment experiments contain solubility tests of ANB powders, where incoming powder defined as $(\mathrm{m} 0)$ when $100 \mathrm{~mL}$ of water within a $250 \mathrm{~mL}$ beaker, followed by magnetically stirring at $750 \mathrm{rpm}$ and room temperature for 1 hour. Insoluble parts (the whole of floated, suspended and deposited) of powders were collected by a filter paper with 4-12 $\mu \mathrm{m}$ pore size and then were dried at $60^{\circ} \mathrm{C}$ for $48-72$ hours and weighed sequentially $\left(m_{1}\right)$ until come to a constant weight. The drying temperature $\left(50^{\circ} \mathrm{C}\right)$ was chosen lower than the degradation temperature of the SA. The amount of water-insoluble powders for surface-treated and untreated ANB powders was noted for each activation time, and then coating yield was evaluated depending on the changes in the powder amounts.

On the other hand, a fraction of feed material other than floated and deposited parts was defined after the solubility test, and noted as a mass loss (\%), indicating its solubility. An attempt was made to have an idea of the coating durability from the weight losses detected at the end of repeated washing processes of
ANB coated with SA. The same procedures were repeated twice in the conditions applied in the solubility test, and the mass loss after the experiment called as triple washing was evaluated.

\section{Results and discussion}

\subsection{Surface morphologies of stearic acid coated and uncoated anhydrous borax}

In Figure 2, SEM images of powders indicate that asreceived ANB samples are angular and mostly individual particles $(\mathrm{a}, \mathrm{c}, \mathrm{e})$, but coated powders with 1 wt. \% SA for $60 \mathrm{~min}$ consists of clustered structures $(b, d, f)$. The SEM images reveal that SA coverage is not well observed at low magnitude on the surfaces of ANB since the percentage of SA is very low. Discrete coating of SA on ANB surface was observed at higher magnifications. Moreover, agglomerated SA particles were not detected. SEM-EDX results $(g, h)$ confirm that existence of SA on the ANB surface as compared to the carbon content of the as-received powders. On the other hand, in the system where the coating process is performed, it is observed that the particles keep their structure morphologies instead of becoming completely spherical. In addition, the largest dimension of the grains was determined to be $105 \mu \mathrm{m}$, which is a result supporting the accuracy of particle size analysis.

\subsection{Influence of the intensity of grinding mechanical forces on the surface modification of anhydrous borax}

Influence of the intensity of grinding mechanical forces on the particle size of ANB powders is quite obvious (Table 2). The particle size of the as-received ANB powders was determined to be under the $500 \mu \mathrm{m}$, and it decreased to less than $100 \mu \mathrm{m}$ after processed for $120 \mathrm{~min}$. On the other hand, the d90 value of coated powders with $1 \mathrm{wt}$ \% SA for $60 \mathrm{~min}$ is lower than that of as-received ANB (Figure. 3). The reduction in particle size of coated ANB was occurred by adding the lubricant host in the grinding medium where the friction between the balls and walls of the jars reduced. Contrary, the $\mathrm{d} 50$ and $\mathrm{d} 10$ values of coated powders are higher than that of as-received ANB powders. This is thought to be due to the fact that stearic acid in the system may cause agglomeration between the fine particles.

The particle size has also a great effect on the solubility of anhydrous borax (Table 3 ). The weight loss, as indicator solubility, of as-received ANB sample in water was determined to be $27.3 \%$. While there is no significant change in weight loss up to the activation time of 60 minutes, this value reaches a level of $40 \%$ after treatment 120 minutes. Furthermore, the weight loss of coated ANB sample $1 \mathrm{wt} \%$ SA for 60 min was decreased to a level of $8.6 \%$. This result indicates that SA particles were considerably bonded to the surfaces of the ANB powders and prevent its solubility in great dual. 

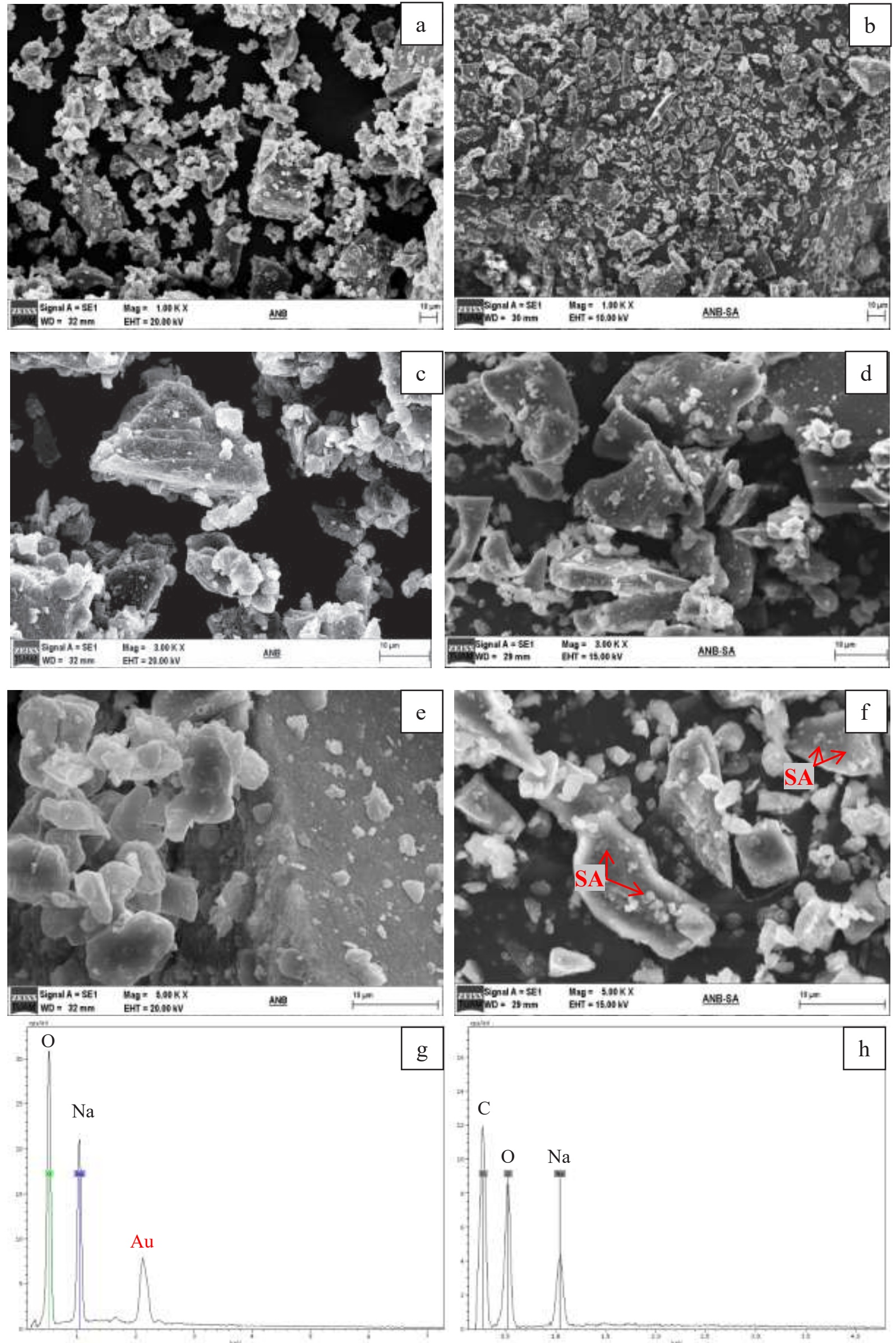

Figure 2. SEM images of as-received and coated ANB powders. 
Table 2. Particle size distribution of as-received ANB powders as a function of activation time.

\begin{tabular}{cccc}
\hline \multirow{2}{*}{ Activation time $(\mathbf{m i n})$} & \multicolumn{3}{c}{ Particle size $(\boldsymbol{\mu m})$} \\
\cline { 2 - 4 } & $\mathbf{d}_{\mathbf{1 0}}$ & $\mathbf{d}_{\mathbf{5 0}}$ & $\mathbf{d}_{\mathbf{9 0}}$ \\
\hline 0 & $86.8 \pm 0.77$ & $267.6 \pm 0.81$ & $499.3 \pm 0.33$ \\
30 & $20.8 \pm 0.43$ & $110.0 \pm 0.84$ & $328.9 \pm 0.49$ \\
60 & $12.4 \pm 0.35$ & $72.3 \pm 0.19$ & $178.6 \pm 0.15$ \\
120 & $6.5 \pm 0.29$ & $40.6 \pm 0.62$ & $100.6 \pm 0.42$ \\
$60^{*}$ & $17.7 \pm 0.16$ & $79.0 \pm 0.34$ & $165.8 \pm 1.39$ \\
\hline
\end{tabular}

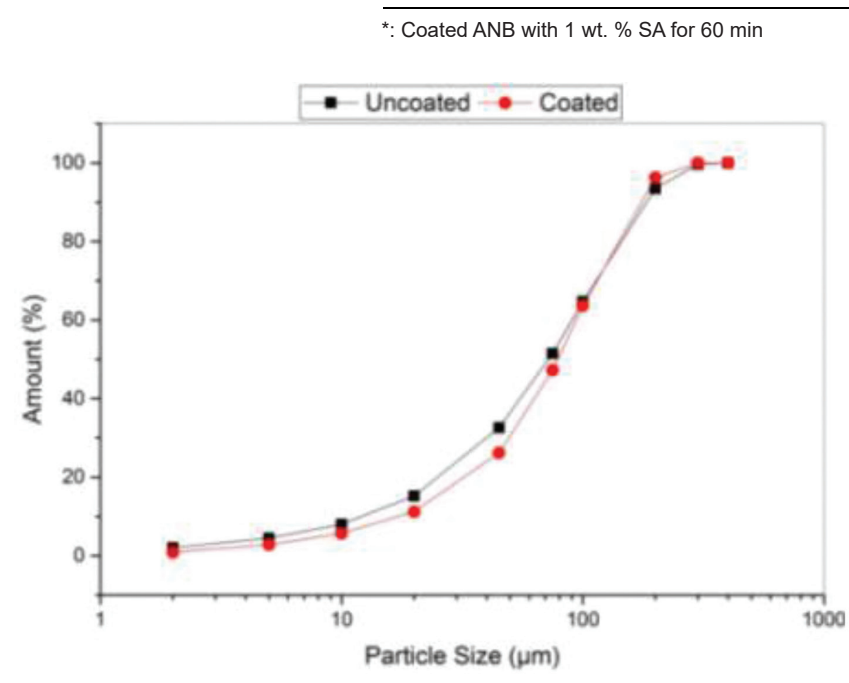

Figure 3. The particle size distribution of as-received and coated ANB powders.

Table 3. Weight loss of ANB in water as a function of activation time.

\begin{tabular}{cc}
\hline $\begin{array}{c}\text { Activation time } \\
\text { (min) }\end{array}$ & $\begin{array}{c}\text { Weight Loss } \\
\text { (wt. \%) }\end{array}$ \\
\hline 0 & 27.3 \\
30 & 25.2 \\
60 & 28.2 \\
120 & 39.7 \\
$60^{*}$ & 8.6 \\
\hline *: Coated ANB with 1 wt. \% SA for 60 min.
\end{tabular}

\subsection{Wettability}

The contact angles of the processed samples at various times were reported in Table 4, as a function of the SA amounts. In order to compare coated samples with pure SA, a disc was prepared and measured as $83.55^{\circ}$. As indicated in the table, the contact angles of the samples were in the range of $17^{\circ}$ to $99.28^{\circ}$ with a standard deviation below $1.1^{\circ}$ and the highest contact angle value obtained was $99.28^{\circ}$ in the coated sample with $1 \mathrm{wt} \% \mathrm{SA}$ for $60 \mathrm{~min}$. High contact angles were observed in all the coated samples that reveal a significant hydrophobic behaviour compared to the raw state. ANB surface could be switched from hydrophilic to hydrophobic with changing contact angle from wetting $17^{\circ}$ to non-wetting $99^{\circ}$, as seen in Figure 4 . On the other hand, it was once stated that stearic acid could cause agglomeration between fine particles in the coating process, and this was observed as an increase in the particle size of ANB powders coated with $1 \mathrm{wt} . \%$ SA. With the further increase in the SA amount (up to $2 \mathrm{wt} . \%)$, this resulted in more agglomeration causing problems such as the adhesion of the powders to the jar wall in the coating medium. Therefore, it is considered that no effective coating was achieved with $2 \mathrm{wt} . \%$ $\mathrm{SA}$, and as a result, a reduction of contact angle values was observed in samples coated with $2 \mathrm{wt} . \%$ SA.

Table 4. Contact angles of as-received and coated-ANB samples.

\begin{tabular}{ccccc}
\hline $\begin{array}{c}\text { Activation } \\
\text { time } \\
\text { (min) }\end{array}$ & \multicolumn{4}{c}{ Amounts of SA (wt.\%) } \\
\cline { 2 - 5 } & $\mathbf{0}$ & $\mathbf{0 . 5}$ & $\mathbf{1 . 0}$ & $\mathbf{2 . 0}$ \\
\hline 30 & $17^{\circ}$ & $96.65^{\circ}$ & $93.63^{\circ}$ & $74.13^{\circ}$ \\
60 & $25^{\circ}$ & $87.73^{\circ}$ & $99.28^{\circ}$ & $87.67^{\circ}$ \\
120 & $27^{\circ}$ & $91.75^{\circ}$ & $97.81^{\circ}$ & $95.65^{\circ}$ \\
\hline
\end{tabular}

\subsection{Evaluation of coating efficiency and durability of stearic acid on anhydrous borax}

In order to determine the insolubility \%, in the evaluation of coating efficiency, as-received and coated ANB samples were analyzed by the water solubility test. Without the treatment by SA, the as-received ANB was partially soluble in water, and the amount of its waterinsoluble part was $73 \%$. When the ANB surface was coated with SA, the water-insoluble part values of all the samples were above $80 \%$ (Table 5 ). The results in the evaluation of the coating efficiency indicate that the hydrophobic organic particles (SA) were partially bonded to ANB. The highest insoluble powder amount to be $91 \%$ was obtained ANB with 1 wt. $\%$ SA coated for $60 \mathrm{~min}$.

When the weight losses after the triple washing of coated ANB powders were evaluated, it was observed that the coating amount of $0.5 \mathrm{wt} . \%$ resulted in more weight loss than that of the others, after the first (1st) washing as seen in Figure 5 . This indicated that the amount of SA ( $0.5 \mathrm{wt} . \%)$ is not sufficient to prevent the water interaction of ANB. The lowest weight loss, depending on solubility, was obtained after coating with 1 wt. \% SA for $60 \mathrm{~min}$. After second (2nd) washing, it was observed that coated ANB powders behaved similarly to those of the first washing. This can be interpreted the coating still maintains its efficiency. Compared with uncoated one, the effect of $1 \mathrm{wt} . \%$ SA coating for 60 min on reducing the solubility of ANB in aqueous media is clearly visible (Table 6). After third (3rd) washing, more than $95 \%$ of ANB powders (for both uncoated and coated with SA at different activation times) were dissolved in water, and this indicated that the 


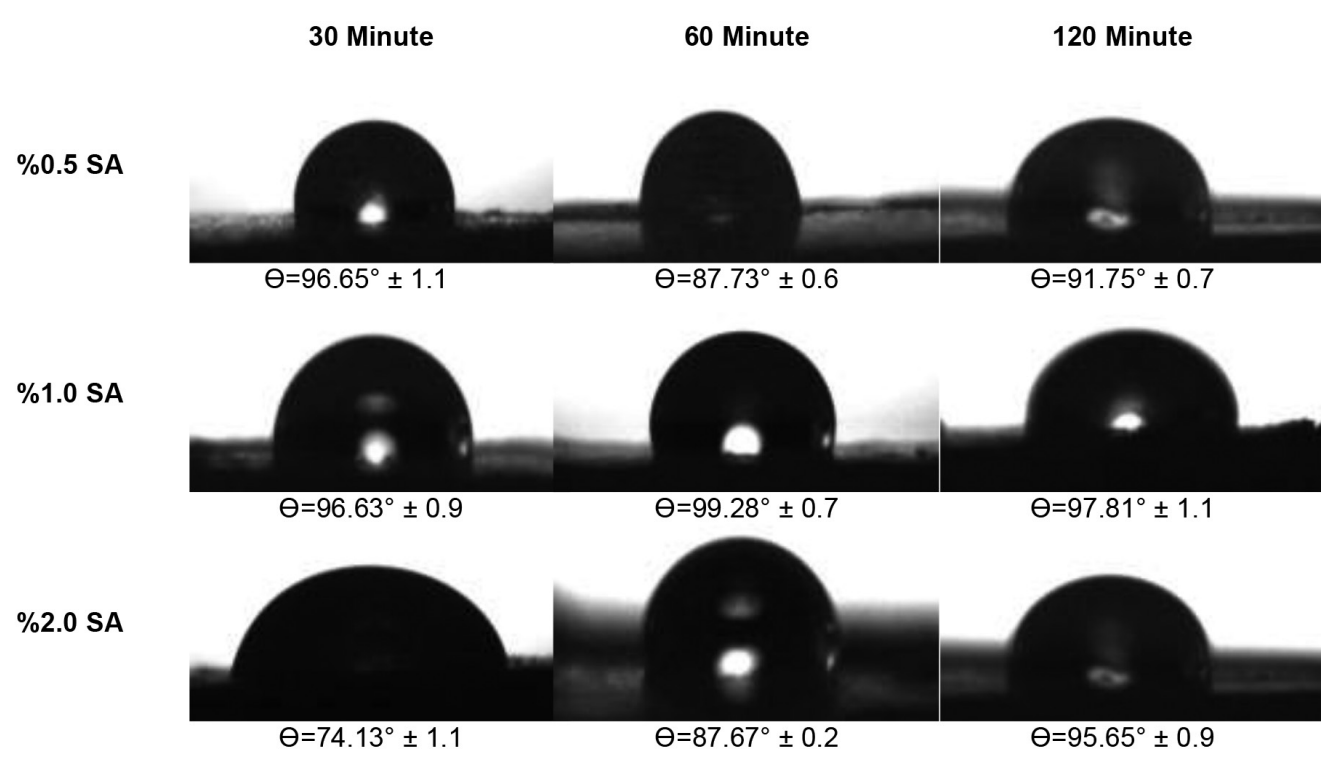

Figure 4. Contact angles of coated ANB samples.

Table 5. Water-insoluble powder values of ANB as a function of activation time.

\begin{tabular}{ccccc}
\hline \multirow{2}{*}{$\begin{array}{c}\text { Activation time } \\
(\boldsymbol{m i n})\end{array}$} & \multicolumn{4}{c}{ Amount of SA (wt. \%) } \\
\cline { 2 - 5 } & $\mathbf{0}$ & $\mathbf{0 . 5}$ & $\mathbf{1}$ & $\mathbf{2}$ \\
\hline 0 & 73 & - & - & - \\
30 & 75 & 84 & 86 & 86 \\
60 & 72 & 87 & 91 & 88 \\
120 & 60 & 78 & 86 & 86 \\
\hline
\end{tabular}

Table 6. Weight loss values after triple washing for as-received and coated ANB.

\begin{tabular}{ccc}
\hline \multirow{2}{*}{$\begin{array}{c}\text { Washing } \\
\text { number }\end{array}$} & \multicolumn{2}{c}{ Solubility (wt.\%) } \\
\cline { 2 - 3 } & As-received ANB & $\begin{array}{c}\text { Coated ANB with } \\
\text { 1 wt. \% SA for 60 min }\end{array}$ \\
\hline $1^{\text {st }}$ & 39.7 & 8.57 \\
$2^{\text {nd }}$ & 76.8 & 49.54 \\
$3^{\text {rd }}$ & 95.3 & 97.57 \\
\hline
\end{tabular}

coating integrity fades away at the conditions of repeated washing.

\section{Conclusions}

Anhydrous borax powder surfaces are highly hydrophilic and could be altered to hydrophobic by using dry coating process and coated with SA, which changed its contact angle from $17^{\circ}$ to $99^{\circ}$. Insoluble powder amount of ANB in water was increased from $73 \%$ to $91 \%$ by coating with $1 \mathrm{wt} . \%$ SA for $60 \mathrm{~min}$. SEM analysis result also demonstrates that $S A$ is mainly coated onto ANB surface. In conclusion, an effective mechanical dry coating processing by a one-step coating approach could be applied to obtain the modified borax surface, which offers controlled solubility behaviour in water-based suspensions. The results obtained avoiding the hydration of anhydrous borax by this surface protection are advantageous for a wide range of water-based applications such as paints, drugs, and suspensions of glazes or ceramics.

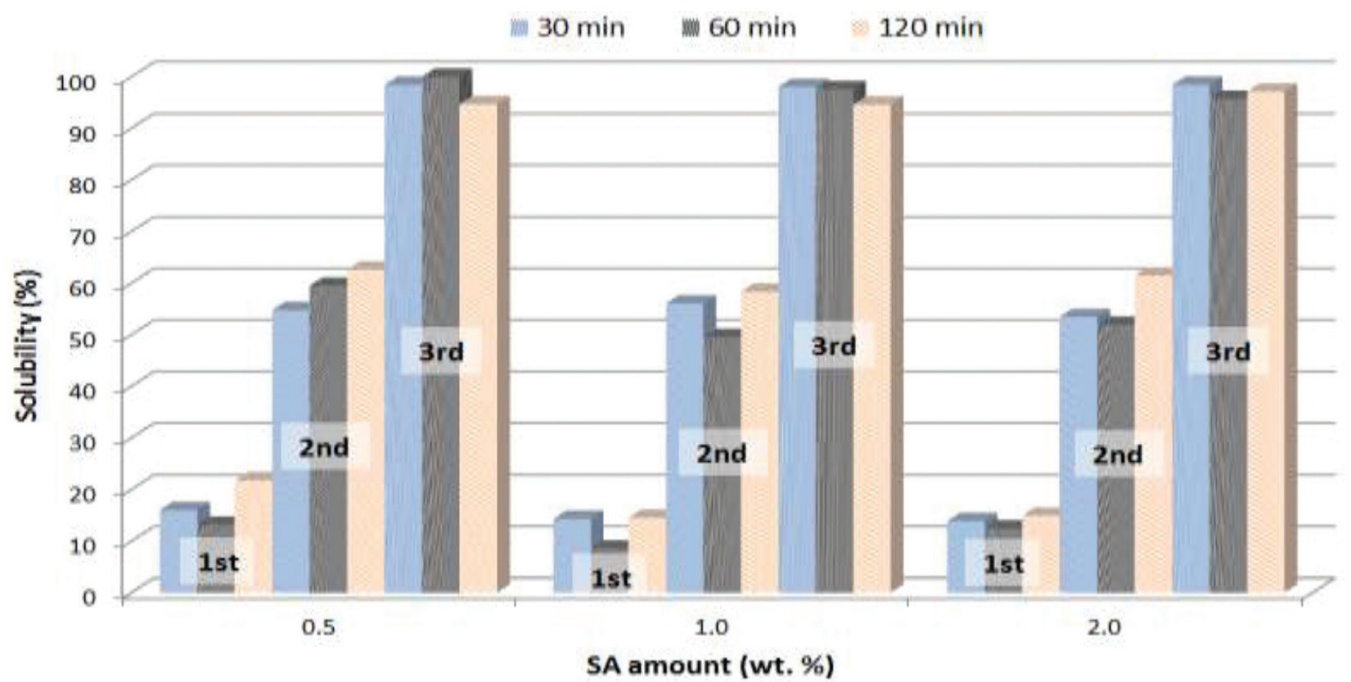

Figure 5. Weight losses of coated ANB powders after triple washing as a function of activation time. 


\section{References}

[1] McMillian P. W., Glass-Ceramics, $2^{\text {nd }}$ Edition, Academic Press, New York, 1979.

[2] Kingery W. D., Bowen H. K., Uhlmann, D. R., Introduction to Ceramics, John Wiley and Sons, New York, 1976.

[3] Hawthorne F. C., Burns P. C., Grice J. D., The crystal chemistry of boron. In Boron Mineralogy, Petrology and Geology (E. S. Grew and L. M. Anevitz, Eds.), Mineral Society American, MSA Reviews in Mineralogy, 33 (2), 41-116, 1996.

[4] Helvaci C. Borates, in Encyclopedia of Geology, (Selley R.C., Cocks, L.R.M and Plimer, I.R., eds.), Elsevier, (3), 510-522, 2005.

[5] Kirk-Othmer, Encyclopedia of Chemical Technology, Ch.4, $5^{\text {th }}$ Edition, 241-294, 2004.

[6] Akpinar S., Yazici Z.O., Can M.F., Investigation of surface-modified anhydrous borax utilisation in raw glazes, Ceram. Int 44, 18344-18351, 2018

[7] ECETOC Technical Report No. 63, Reproductive and General Toxicology of Some Inorganic Borates and Risks Assessment for Human Beings, European Centre for Ecotoxicology and Toxicology of Chemicals (ECETOC), Brussels, Belgium, 3, 1995.

[8] Kaplan J., Zamek J., A substitute for gerstley borate, Ceramics Technical, 32, 24-29, 2011.

[9] Otles M. S., Modification of surface properties of biopowders by dry particle coating, Ph.D. Thesis, Université de Toulouse, National Institute of Posts and Telecommunications, Rabat, 2008.

[10] Pfeffer R., Dave R. N., Dongguang W., Ramlakhan M., Synthesis of engineered particulates with tailored properties using dry particle coating, Powder Technol., 117, pp.40-67, 2001.

[11] Yoshihara I., Pieper W., Hybridization-technology for surface modification of powders without binders, Swiss Pharma 6, 21, 1999.

[12] Ouabbas Y., Chamayou A., Galet L., Baron M., Thomas G., Grosseau P., Guilhot B., Surface modification of silica particles by dry coating: Characterization and powder ageing, Powder Technol., 190a, 200-209, 2009.

[13] Lefebvre G., Galet L., Chamayou A., Dry coating of talc particles: Effect of material and process modifications on their wettability and dispersibility, AIChE Journal, 57 (1), 79-86, 2011.

[14] Sonoda R., Horibe M., Oshima T., Iwasaki T., Watano S., Improvement of dissolution property of poorly water-soluble drug by novel dry coating method using planetary ball mill, Chem. Pharm. Bull. 56 (9),1243-7, 2008.

[15] Tsai W. T., Microstructural characterization of calcitebased powder materials prepared by planetary ball milling, Materials, 6, 3361-3372, 2013.
[16] Suryanarayana C., Mechanical alloying and milling, Prog. Mater Sci., 46, 1-184, 2001.

[17] Baláž P., Mechanochemistry in Nanoscience and Minerals Engineering, Chapter 2, High Energy Milling, Springer, Hardcover, Netherland, 2008.

[18] Hersey J. A., Ordered mixing: A new concept in powder mixing practice, Powder Technology, 11 (1) 41-44, 1975.

[19] Dahmash J., Dry particle coating-a unique solution for pharmaceutical formulation, Pharmaceutical Technology, 42 (3) 26-30, 2018.

[20] Gilbert M., Petiraksakul P., Mathieson I., Characterization of stearate-stearic acid coated fillers, Mater. Sci. Technol., (17), 1472-1478, 2001.

[21] Jeong S. B., Yang, Y. C., Chae Y.B., Kim B. G., Characteristics of the treated ground calcium carbonate powder with stearic acid using the dry process coating system, Mater. Trans., 50 (2), 409-414, 2009.

[22] Mihajlovic S. R., Vucinic D. R., Sekulic Z. T., Milicevic S. Z., Kolonja B. M., Mechanism of stearic acid adsorption to calcite, Powder Technol., 245, 208-216, 2013.

[23] Gilbert M., Sutherland I., Guest A., Characterization of coated particulate fillers, J. Mater. Sci., 35, 391-397, 2000.

[24] Griffin M., The Structure and Biological Functions of Lipids, Bio Factsheets, 3, 2000.

[25] Markley K. S., Fatly Acids: Their chemistry and physical properties, Part 1, $2^{\text {nd }}$ ed., Interscience Publishers Inc., New York. 1960.

[26] Agernäs O., Tengberg T., Development of two methods to evaluate lubricating greases using a rheometer, B.Sc. Thesis in Chemical Engineering, Chalmers University of Technology, Göteborg, Sweden, 2011.

[27] Hashmi S., Effect of surface roughness on wetting properties, in Comprehensive Materials Finishing, 285, 2017.

[28] Kanimozhi D., Ratha Bai V., Analysis of bioactive components of ethanolic extract of coriandrum sativum L., IJRPS 2012, 2 (3),97-110, 2012.

[29] Jeong S. B., Yang Y. C., Chae Y. B., Kim B. G., Characteristics of the treated ground calcium carbonate powder with stearic acid using the dry process coating system, Mater. Trans., (50-2), 409 - 414, 2009.

[30] Liao J., Du G., Qiao X., Hao D., Surface modification of diatomite by stearic acid and its effects on reinforcing for natural rubber/styrene-butadiene rubber blend. J. Chin. Ceram Soc., (39), 641-645, 2011.

[31] Wang Y., Lee W. C., Characterization and treatment of calcium carbonate: A comparative study, Polym. Compos., (24), 119-131, 2003.

[32] Wang B. B., Feng J. T., Zhao Y. P., Yu T. X., Fabrication of novel superhydrophobic surfaces and water droplet bouncing behavior, J. Adhes. Sci. Technol., 24, 26932705, 2010. 